\title{
OBSERVATIONS OF ATOMIC HYDROGEN IN \\ DENSE INTERSTELLAR DUST CLOUDS
}

\author{
GILLIAN R. KNAPP \\ University of Maryland, Md., U.S.A.
}

\begin{abstract}
High frequency resolution $21-\mathrm{cm}$ line observations of almost 100 interstellar dust clouds showed that about one-third of them contain significant amounts of cold neutral hydrogen. Simple models for the velocity distribution of hydrogen in the clouds were fitted to the observations, and spin temperatures ranging from 16 to $40 \mathrm{~K}$ and column densities from $7 \times 10^{19}$ to $6 \times 10^{20}$ atom $\mathrm{cm}^{-2}$ were found. The ratios of $\mathrm{H}$ I to $\mathrm{OH}$ and $\mathrm{H}$ I to dust in the clouds were found to have about 1 to $10 \%$ of their value in the general interstellar medium.
\end{abstract}

Most dense interstellar dust clouds have not been detected as excess emission in neutral hydrogen, leading to the speculation that most of the hydrogen in such clouds is molecular. If, however, the $\mathrm{HI}$ in these clouds is colder than that in the surrounding interstellar medium, it should be detectable as narrow self-absorption features in $\mathrm{H} \mathrm{I}$ profiles in the directions of such clouds, assuming that the cold dust cloud is in front of most of the rest of the hydrogen, which then acts as a background to be absorbed. Observations of profiles in the directions of almost 100 dense interstellar dust clouds made with the NRAO 140-ft telescope using high velocity resolution (0.34 and 0.17 $\mathrm{km} \mathrm{s}^{-1}$ ) showed the presence of self-absorption dips for about one-third of the clouds observed (Knapp, 1972). These dips are very narrow (half-widths from 0.9 to 4.5 $\left.\mathrm{km} \mathrm{s}^{-1}\right)$, are at the same velocities as the molecular lines observed for the clouds, and are most likely to be observed for fairly dark clouds $\left(A_{v} \geqslant 3^{m}\right)$. A sample profile for the large cloud Kahvtassi 141 is shown in Figure 1.

The observations were analysed to find the neutral hydrogen content of the dust clouds assuming that the gas in each cloud has a gaussian dependence on velocity and a constant spin temperature, and that there is no filling-up of the self-absorption dip by emission from $\mathrm{H} \mathrm{I}$ in front of the dust cloud. The solutions indicate spin temperatures from 16 to $40 \mathrm{~K}$ (which are higher values than those found for the molecules), peak optical depths from about 0.5 to 4.0 , and column densities from $7 \times 10^{19}$ to $6 \times 10^{20}$ atom $\mathrm{cm}^{-2}$. These values are about $1-10 \%$ of those predicted from the observed $\mathrm{OH}$ and dust column densities, so that the rest of the hydrogen is presumably in molecular form. Using optical and kinematic distance estimates, values for the neutral hydrogen number densities ranging from 3 to 77 atom $\mathrm{cm}^{-3}$ were derived for eleven clouds.

The kinematics of the whole sample of dust clouds was examined using these velocities and distances, and it was found that most of the dust clouds belong to the local Gould Belt expanding system, in which they are embedded more or less at random. The rest of the clouds belong to the other local structural feature, the Orion arm. 
The fact that $\mathrm{H}_{2} \mathrm{CO}, \mathrm{OH}, \mathrm{CO}$ and $\mathrm{H}$ I observations for the same cloud often have roughly the same line widths strongly suggests the presence of systematic expansion or contraction motions. The $\mathrm{H}$ I line widths are often slightly larger than those for

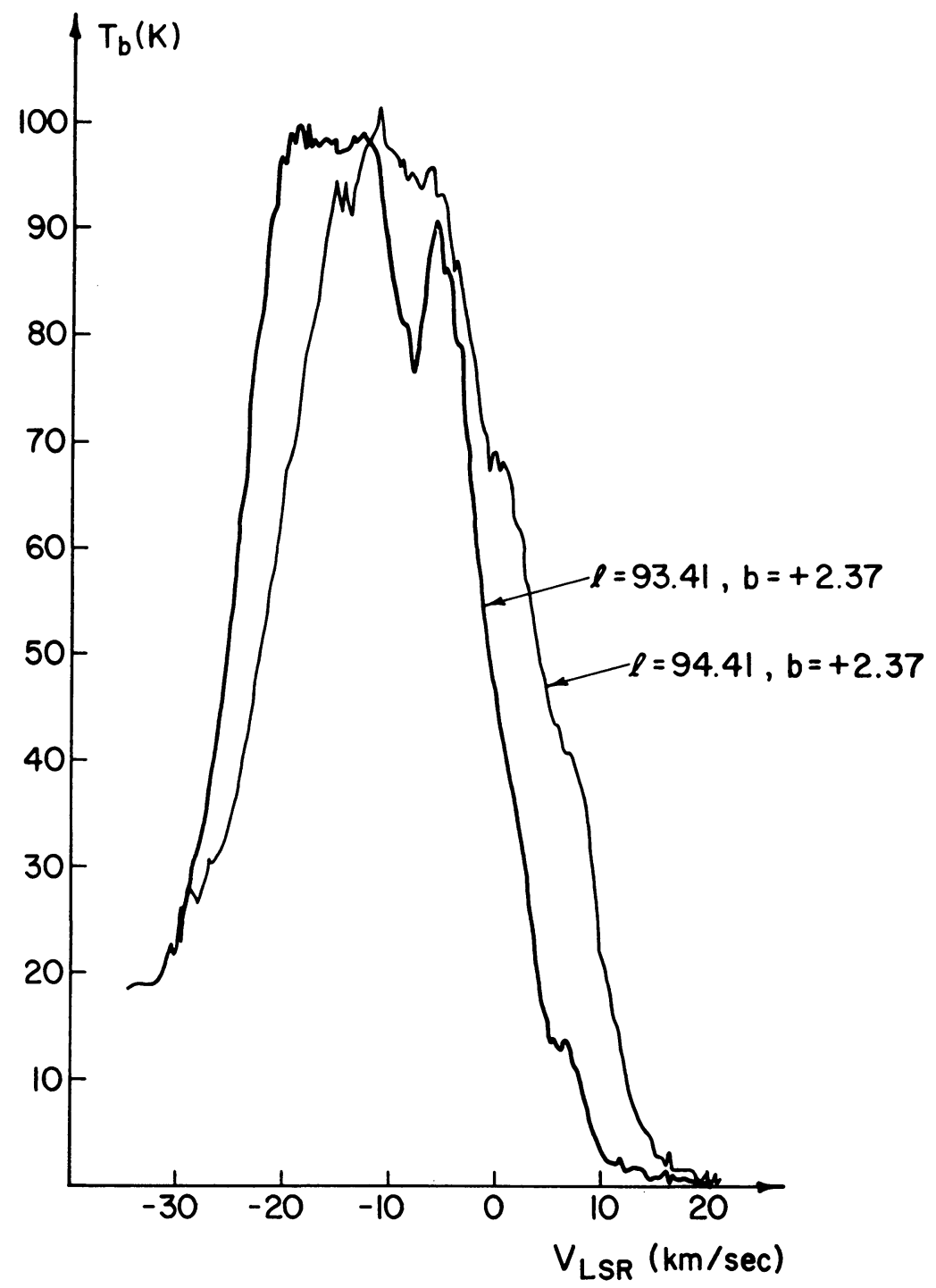

Fig. 1. Sample $\mathrm{H}$ I profiles in the direction of $\mathrm{Kh} \mathrm{141.} \mathrm{The} \mathrm{'on-cloud'} \mathrm{profile} \mathrm{is} \mathrm{drawn} \mathrm{with} \mathrm{a} \mathrm{heavy}$ line, and the 'off-cloud' (comparison) profile with a lighter line.

the molecules, indicating that the $\mathrm{H}$ I distribution extends slightly outside that of the molecules. The $\mathrm{H}$ I self-absorption profiles for several clouds show structure suggesting the presence of temperature gradients and expansion/contraction motions (with 
velocities of about $1 \mathrm{~km} \mathrm{~s}^{-1}$ ), and analysis of simple models shows that, if these clouds are contracting, they are slightly hotter in the center than on the outside.

\section{Reference}

Knapp, G. R.: 1972, Ph.D. Thesis, University of Maryland. 